\title{
Intellectual Disability and Hidradenitis Suppurativa
}

\author{
Elia Rosi Maria Thais Fastame Leonardo Pescitelli Antonella Di Cesare \\ Federica Ricceri Nicola Pimpinelli Francesca Prignano
}

Section of Dermatology, Department of Health Sciences, University of Florence, Florence, Italy

\section{Keywords}

Hidradenitis suppurativa · Intellectual disability ·

Comorbidities

\section{Dear Editor,}

Hidradenitis suppurativa (HS) is a chronic, inflammatory disorder that affects apocrine gland-bearing skin clinically characterized by recurrent painful deep-seated nodules, abscesses, sinus tracts, bridge scars and tombstone open comedones [1].

Quality of life in HS is lower than that of several other chronic dermatological conditions [2].

Indeed, HS patients suffer from numerous other comorbidities, and emerging evidence suggests the association of HS with mental disorders $[2,3]$. The burden of mental health conditions may further worsen the already poor quality of life of people affected by HS.

Intellectual disability (intellectual developmental disorder) (ID), formerly known as mental retardation, is characterized by significantly impaired cognitive functions [4]. Different ID definitions exist in the literature; the Diagnostic and Statistical Manual of Mental Disorders (DSM-5) defines ID as a disorder that occurs during the period of development, characterized by intellectual and adaptive deficits [4]. ID severity, in more than $75 \%$ of individuals, is mild, and, in these patients, an underlying specific aetiology is less likely to be identified [5].

Since, to the best of our knowledge, there are no previous data, we performed a retrospective chart review in order to investigate the association between HS and ID (not due to an underlying specific cause) and to assess whether the concomitant presence of ID could affect the HS phenotype.

Demographic and clinical data of all consecutive patients with HS attending our tertiary dermatology outpatient clinic, between March 1, 2018, and March 1, 2019, were retrospectively retrieved and analysed. Patients were considered affected by ID only if the diagnosis was documented by a mental health physician who excluded a specific ID aetiology (such as genetic abnormalities, inborn errors of metabolism and brain malformations).

We identified a total of 112 patients with HS (40 males, $35.7 \%$, and 72 females, $64.3 \%$ ) seen during this time. Demographic and clinical characteristics of HS patients are shown in Table 1.

Seven patients out of 112 (6.2\%) had concomitant HS and ID. ID severity was mild in all patients. A family history of HS was reported in 9/112 (8\%) cases; notably, karger@karger.com

(c) 2020 S. Karger AG, Basel

www.karger.com/drm

Karger ${ }^{\prime}=$
Francesca Prignano

Section of Dermatology, Department of Health Sciences, University of Florence Ospedale P. Palagi, viale Michelangiolo, 41

IT-50125 Florence (Italy)

francesca.prignano@unifi.it 
Table 1. Demographic and clinical characteristics of HS patients

\begin{tabular}{lccc}
\hline & $\begin{array}{l}\text { HS only } \\
(n=105)\end{array}$ & $\begin{array}{l}\text { HS + ID } \\
(n=7)\end{array}$ & $p$ value \\
& $36(34.3)$ & $4(57.1)$ & $0.246^{\mathrm{a}}$ \\
Male, $n(\%)$ & $69(65.7)$ & $3(42.9)$ & \\
Female, $n(\%)$ & & & \\
Age (mean \pm SD), years & & & \\
$\quad$ At onset & $26.2(9.8)$ & $15.1(5.5)$ & $0.002^{\mathrm{c}}$ \\
$\quad$ At diagnosis & $32.6(12.9)$ & $20.6(6.6)$ & $0.015^{\mathrm{c}}$ \\
BMI (mean \pm SD) & $25.8(5.2)$ & $26.9(5.5)$ & $0.496^{\mathrm{c}}$ \\
Hurley stage, $n(\%)$ & & & \\
$\quad$ I & $39(37.1)$ & $1(14.3)$ & $0.418^{\mathrm{a}}$ \\
$\quad$ II & $42(40)$ & $5(71.4)$ & $0.128^{\mathrm{a}}$ \\
$\quad$ III & $24(22.9)$ & $1(14.3)$ & $1.000^{\mathrm{a}}$ \\
Smokers, $n(\%)$ & $72(68.6)$ & $1(14.3)$ & $0.007^{\mathrm{a}}$ \\
Involved areas, $n(\%)$ & $57(54.3)$ & $3(42.8)$ & $0.703^{\mathrm{a}}$ \\
$\quad$ Groin & $39(37.1)$ & $6(85.7)$ & $0.011^{\mathrm{b}}$ \\
$\quad$ Axillae & $12(11.4)$ & $1(14.3)$ & $0.589^{\mathrm{a}}$ \\
$\quad$ Pubic region & $9(8.6)$ & $0(0)$ & $1.000^{\mathrm{a}}$ \\
$\quad$ Buttocks & $9(8.6)$ & $0(0)$ & $1.000^{\mathrm{a}}$ \\
$\quad$ Abdomen/chest & & & \\
Current therapies, $n(\%)$ & $48(45.7)$ & $2(28.6)$ & $0.458^{\mathrm{a}}$ \\
$\quad$ Topical antibiotics & $30(28.6)$ & $3(42.8)$ & $0.418^{\mathrm{a}}$ \\
Systemic antibiotics & $27(25.7)$ & $2(28.6)$ & $1.000^{\mathrm{a}}$ \\
$\quad$ Biologics & & & \\
\hline
\end{tabular}

Statistically significant $p$ values are shown in italics. BMI, body mass index. ${ }^{\text {a }}$ Fisher exact test. ${ }^{b} \chi^{2}$ test. ${ }^{c}$ Wilcoxon rank-sum (Mann-Whitney) test.

none of the patients with ID had an HS-positive family anamnesis. Most patients with HS and concomitant ID had a moderate disease severity (Hurley stage I: 1 patient; Hurley stage II: 5 patients; Hurley stage III: 1 patient). Except for 1 patient who suffered from anxiety, no other comorbidities were recorded in the HS + ID group.

The mean age of HS onset was significantly earlier in patients who suffered from HS + ID than in those with HS only (mean age \pm SD: $15.1 \pm 5.5$ vs. $26.2 \pm 9.8$ years; $p<0.01$ ), and patients with concomitant ID were younger at HS diagnosis (mean age \pm SD: $20.6 \pm 6.6$ vs. $32.6 \pm$ 12.9 years; $p<0.05$ ) compared to HS-only patients (shown in Table 1). Delay in diagnosis was similar in the two groups. Characteristic clinical manifestations of HS are preferentially located in the axillary area in patients with associated ID. In our case series, the smoker percentage was significantly lower in the HS + ID group compared to the HS-only group ( 14.3 vs. $68.6 \%$; $p<0.01$ ).

No statistically significant differences were found between HS and HS + ID groups regarding gender, body mass index, disease severity and current therapies (Table 1).

Intellectual Disability and Hidradenitis Suppurativa
Taken together, our data suggest that HS may be associated with ID with an earlier presentation/age of onset of typical HS cutaneous lesions. This finding is in line with that of other authors $[6,7]$ who found a younger age of HS onset in patients with Down syndrome (DS), the most frequent genetic cause of ID, compared to those with HS only (14.9 vs. 23.3 years [6] and 14.3 vs. 23.4 years [7]). In our study, the mean age at onset of HS symptoms (in patients with concomitant ID) was 15.1 years, which supports this observation. Furthermore, in our study, the occurrence of ID in patients with HS was $6.2 \%$, at least twice that of the general population (often quoted at $1 \%$ globally, but variable from 1 to $3 \%$ by country, according to the heterogeneity in study settings and case definitions [5]). Interestingly, the same authors mentioned above detected that the prevalence of DS (in the evaluated cohorts) is higher in HS patients compared to the general population $(2.4 \%$ [6] and $3.5 \%$ [7] vs. $0.083 \%$ in the general population). However, in our study, the overall sample size is too small to extrapolate prevalence data, and population-based studies are needed. Moreover, as it has been assessed for DS $[8,9]$, it would be more interesting, in our opinion, to determine the prevalence of HS among patients with ID, and not vice versa. Indeed, a confirmed high prevalence of HS in the population affected by ID would mean a greater awareness of the possible increased risk of developing HS in those patients with ID (with implications in the surveillance of these "fragile" patients). On the other hand, in the rare cases in which ID diagnosis by a mental health physician is not already present, dermatologists should suspect ID in subjects who have difficulty in the comprehension of complex language, who need assistance in making health care decisions and whose behaviour in social situations may be considered immature.

Imbalance in the regulation of cytokines could represent the pathophysiological basis of this association. Indeed, pro-inflammatory cytokines (such as tumour necrosis factor, interleukin (IL)-1 and IL-6) have been correlated with several neurodevelopmental disorders, and their multiple functions in the central nervous system suggest their central role in complex central nervous system functions such as cognition. According to this perspective, Aureli et al. [10] supported the hypothesis that IL-6 may play an important role in the development of ID (suggesting that the IL-6 and IL-1RA polymorphisms as well as the overexpression of IL-6 might have significant implications in ID pathogenesis). On the other hand, the involvement of a variety of cytokines such as tumour necrosis factor- $\alpha$, IL-1 $\beta$, IL-10 and IL- 6 in HS pathogenesis is well known [11]. 
Concerning smoking habits, in our opinion, the lower percentage of smokers in the HS + ID group (compared to the HS-only group) may have to do with the influence that concerned family members have on smoking rates; indeed, the place of residence is highly predictive of smoking status in individuals with ID (those living in institutions or family settings smoke less than those living more independently in the community) [12]. In line with this hypothesis, all our ID patients live with their family members who take care of them.

In conclusion, our results were in line with those reported previously for patients with concomitant HS and DS. The novelty of our study lies in the fact that we have considered ID not due to an underlying specific cause (such as DS), assessing its influence on the HS phenotype (presentation/age of onset/location of typical HS lesions and smoking habits).

The high psychosocial burden of both conditions, the possible common immunological pathways and the possible interference of ID in the adherence to HS medications should be taken into account when treating those patients.

\section{Key Message}

The concomitant presence of intellectual disability may affect the phenotype of hidradenitis suppurativa.

\section{Statement of Ethics}

Ethical clearance was obtained from the local ethics committee.

\section{Disclosure Statement}

The authors have no conflicts of interest to declare.

\section{Funding Sources}

There are no funders to report for this submission.

\section{Author Contributions}

All authors contributed equally to this paper.

\section{References}

1 Ingram JR. Hidradenitis suppurativa: an update. Clin Med (Lond). 2016 Feb;16(1):70-3.

2 Patel ZS, Hoffman LK, Buse DC, Grinberg AS, Afifi L, Cohen SR, et al. Pain, psychological comorbidities, disability and impaired quality of life in hidradenitis suppurativa. Curr Pain Headache Rep. 2017;21(12):49.

3 Fimmel S, Zouboulis CC. Comorbidities of hidradenitis suppurativa (acne inversa). Dermatoendocrinol. 2010 Jan;2(1):9-16.

4 McKenzie K, Milton M, Smith G, OuelletteKuntz H. Systematic review of the prevalence and incidence of intellectual disabilities: current trends and issues. Curr Dev Disord Rep. 2016;3(2):104-15.

5 Patel DR, Cabral MD, Ho A, Merrick J. A clinical primer on intellectual disability. Transl Pediatr. 2020 Feb;9(S1 Suppl 1):S23-35.
6 Denny G, Anadkat MJ. Hidradenitis suppurativa (HS) and Down syndrome (DS): increased prevalence and a younger age of hidradenitis symptom onset. J Am Acad Dermatol. 2016 Sep;75(3):632-4.

7 Giovanardi G, Chiricozzi A, Bianchi L, De Simone C, Dini V, Franceschini C, et al. Hidradenitis Suppurativa Associated with Down Syndrome Is Characterized by Early Age at Diagnosis. Dermatology. 2018;234(12):66-70.

8 Garg A, Strunk A, Midura M, Papagermanos V, Pomerantz H. Prevalence of hidradenitis suppurativa among patients with Down syndrome: a population-based cross-sectional analysis. Br J Dermatol. 2018 Mar;178(3): 697-703.
9 Sechi A, Guglielmo A, Patrizi A, Savoia F, Cocchi G, Leuzzi M, et al. Disseminate recurrent folliculitis and hidradenitis suppurativa are associated conditions: results from a retrospective study of 131 patients with Down syndrome and a cohort of 12,351 pediatric controls. Dermatol Pract Concept. 2019;9(3): 187-94.

10 Aureli A, Sebastiani P, Del Beato T, Marimpietri AE, Graziani A, Sechi E, et al. Involvement of IL-6 and IL-1 receptor antagonist on intellectual disability. Immunol Lett. 2014 Nov;162(1 1 Pt A):124-31

$11 \mathrm{Xu} \mathrm{H}$, Xiao X, He Y, Zhang X, Li C, Mao Q, et al. Increased serum interleukin-6 levels in patients with hidradenitis suppurativa. Postepy Dermatol Alergol. 2017 Feb;34(1):82-4.

12 Steinberg ML, Heimlich L, Williams JM. Tobacco use among individuals with intellectual or developmental disabilities: a brief review. Intellect Dev Disabil. 2009 Jun;47(3):197207. 